Studies of highly charged iron ions using electron beam ion traps for interpreting astrophysical spectra

This content has been downloaded from IOPscience. Please scroll down to see the full text. 2013 Phys. Scr. 2013014001

(http://iopscience.iop.org/1402-4896/2013/T156/014001)

View the table of contents for this issue, or go to the journal homepage for more

Download details:

IP Address: 134.76.223.157

This content was downloaded on 07/05/2014 at 16:08

Please note that terms and conditions apply. 


\title{
Studies of highly charged iron ions using electron beam ion traps for interpreting astrophysical spectra
}

\author{
G V Brown ${ }^{1}$, C Beilmann ${ }^{2}$, S Bernitt ${ }^{2}$, J Clementson ${ }^{1}$, S Eberle ${ }^{2}$, \\ S W Epp ${ }^{3}$, A Graf ${ }^{1}$, N Hell $^{4}$, R L Kelley ${ }^{5}$, C A Kilbourne ${ }^{5}$, K Kubiček ${ }^{2}$, \\ M A Leutenegger ${ }^{5,6}$, V Mäckel $^{2}$, F S Porter ${ }^{5}$, J K Rudolph ${ }^{2,7}$, M C Simon ${ }^{8}$, \\ R Steinbrügge $^{2}$, E Träbert $^{1}$, J Ullrich ${ }^{2,9}$, J R Crespo López-Urrutia ${ }^{2}$ \\ and $P$ Beiersdorfer ${ }^{1}$

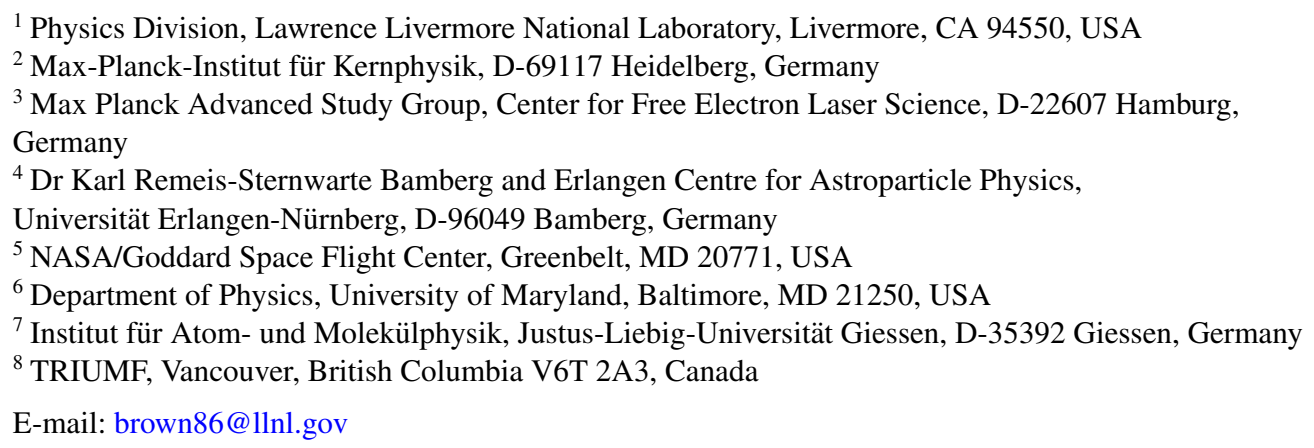

Received 3 September 2012

Accepted for publication 31 October 2012

Published 23 September 2013

Online at stacks.iop.org/PhysScr/T156/014001

\begin{abstract}
For over a decade, the x-ray astrophysics community has enjoyed a fruitful epoch of discovery largely as a result of the successful launch and operation of the high resolution, high sensitivity spectrometers on board the Chandra, XMM-Newton and Suzaku X-ray observatories. With the launch of the X-ray calorimeter spectrometer on the Astro-H x-ray observatory in 2014, the diagnostic power of high resolution spectroscopy will be extended to some of the hottest, largest and most exotic objects in our Universe. The diagnostic utility of these spectrometers is directly coupled to, and often limited by, our understanding of the x-ray production mechanisms associated with the highly charged ions present in the astrophysical source. To provide reliable benchmarks of theoretical calculations and to address specific problems facing the $\mathrm{x}$-ray astrophysics community, electron beam ion traps have been used in laboratory astrophysics experiments to study the x-ray signatures of highly charged ions. A brief overview of the EBIT-I electron beam ion trap operated at Lawrence Livermore National Laboratory and the Max-Planck-Institut für Kernphysik's FLASH-EBIT operated at third and fourth generation advanced light sources, including a discussion of some of the results are presented.
\end{abstract}

PACS numbers: 32.30.Rj, 31.15.ag, 32.70.Fw

(Some figures may appear in color only in the online journal)

\section{Introduction}

Beginning with the successful launches of the Chandra and XMM-Newton X-ray observatories in 1999 and 2000,

${ }^{9}$ Now at Physikalisch-Technische Bundesanstalt, D-38116 Braunschweig, Germany. respectively, high energy astrophysics entered a new era of discovery space. The strong, unresolved Fe-L shell emission features measured earlier in many objects provided the driving force for designing the high resolution grating spectrometers flown on the Chandra and XMM-Newton X-ray observatories and as a result these gratings have effective areas that 


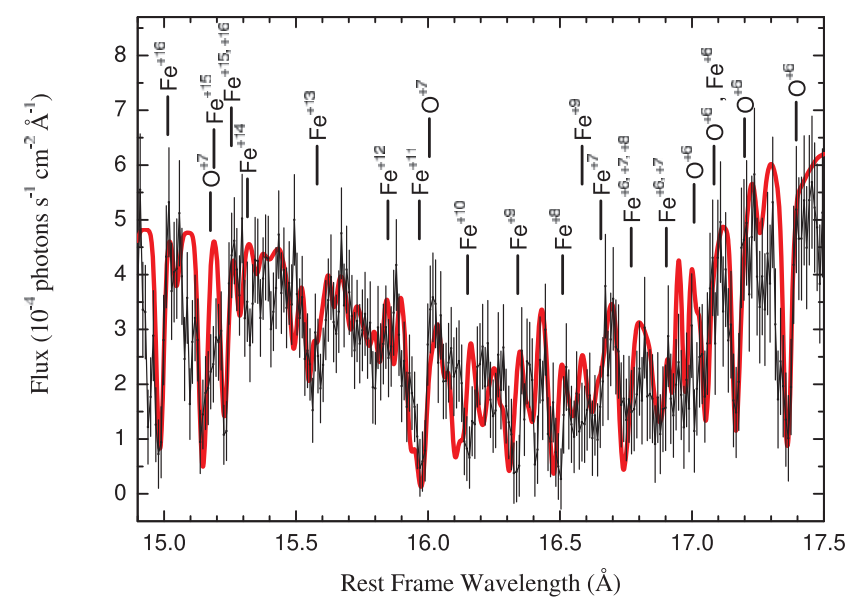

Figure 1. Chandra high energy transmission grating spectrum of active galactic nuclei NGC 3783 poorly fit with a model where the velocity of the $\mathrm{Fe}^{15+}$ ions are at the same velocity as the highly charged oxygen and $\mathrm{Fe}^{16+}$ ions [10]. Gu et al [11] later found a much better fit to this spectrum using an improved $\mathrm{Fe}^{15+}$ atomic data and found that all the ions are moving at $-590 \mathrm{~km} \mathrm{~s}^{-1}$. The discrepancy between theoretical models demonstrated the need for more laboratory measurements. Figure is reproduced with permission from [10].

peak around $15 \AA$, i.e. near the center of the strongest Fe L-shell emission. In addition, both satellites have significant collection areas in the $\mathrm{Fe}-\mathrm{K}$ band, although the resolution is significantly lower than at Fe-L. Chandra transmission gratings and XMM-Newton's reflection grating spectrometer have produced some spectacular high resolution spectra in both absorption and emission (e.g. see the Chandra spectrum in figure 1), however, the high resolution spectra of this type are limited to point sources or sources with rather small angular extent. The launch of Astro-H [1] in 2014 will for the first time bring high resolution spectroscopy to extended sources. Astro-H's soft x-ray spectrometer, which consists of a soft $\mathrm{x}$-ray telescope and the $\mathrm{x}$-ray calorimeter spectrometer [2, 3], has an energy resolution of $\sim 5 \mathrm{eV}$ and effective area of $\sim 200 \mathrm{~cm}^{2}$ at $7 \mathrm{keV}$. Once Astro-H is in flight, the three orbiting $\mathrm{X}$-ray observatories will make it possible to measure high resolution spectra from nearly every strong source in the $\mathrm{x}$-ray sky.

The ability to decode the spectra provided by these observatories requires a thorough understanding of the underlying atomic physics taking place in a variety of highly charged ions. Laboratory astrophysics [4] experiments have played a significant role in furthering our understanding of atomic physics and addressing specific problems facing the astrophysics community. In addition to laboratory data, decoding x-ray spectra also requires well tested spectral models that can extend the results covered by experiments over the entire phase space necessary to interpret astrophysical spectra. This synergistic approach has driven both theory and experiment to provide complete sets of highly accurate, well understood atomic data. Both theory and experiment, however, are constantly being pressed by novel high resolution, high sensitivity instruments on $\mathrm{x}$-ray observatories.

\section{EBIT-I and the FLASH-EBIT}

Electron beam ion trap (EBIT)-I, and more recently, the FLASH-EBIT have been used to study the atomic processes

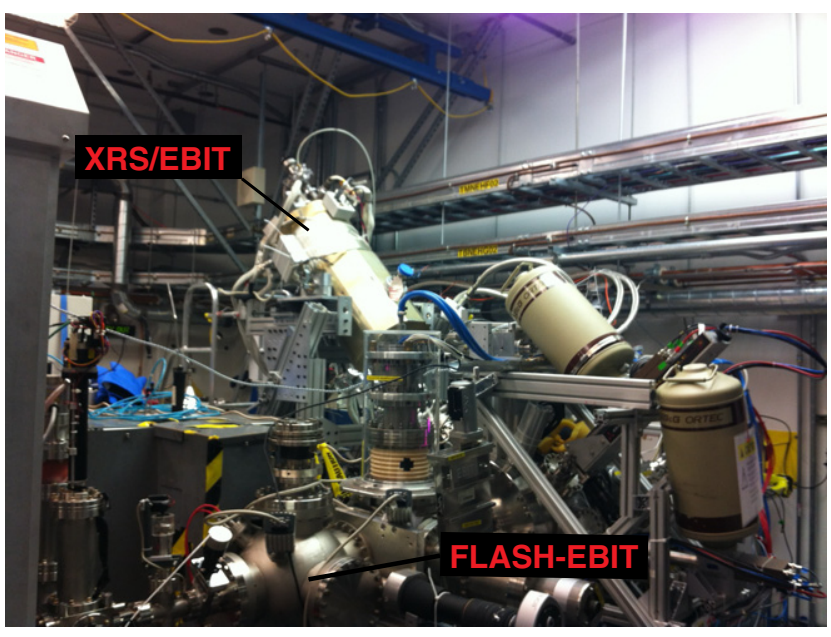

Figure 2. Photograph of the experimental setup of the FLASH-EBIT in the soft $\mathrm{x}$-ray hutch at the linac coherent light source (LCLS).

in astrophysically relevant highly charged ions. EBIT-I has been in operation for several decades and has been extensively described elsewhere (see [4, 5] and references therein). It currently houses a large suite of spectrometers that span the wavelength band from the hard $\mathrm{x}$-ray to the optical. This suite includes several grating spectrometers, crystal spectrometers, solid state detectors and the EBIT calorimeter spectrometer (ECS) [6, 7]. Typical arrangements during experiments include a grating spectrometer, a crystal spectrometer, a solid state detector and the ECS. This provides the means for measuring several wavelength bands simultaneously. In addition to the suite of spectrometers, several modes of operation have been developed at the Lawrence Livermore National Laboratory (LLNL) facility, including the magnetic trapping mode [8] and the event mode [9], that have made it possible to study and isolate a variety of atomic processes.

The EBIT-I electron beam ion trap has been used to address a number of astrophysics problems in several sources including, but not limited to, stellar coronae including the Sun [12, 13], comets [14], planetary atmospheres [15] and high mass x-ray binaries [16]. In addition, measurements from the LLNL's EBITs have been used to benchmark atomic physics codes and spectral models used to interpret spectra from nearly every high energy astrophysical source.

While EBIT-I experiments have focused on addressing laboratory astrophysics problems by studying emission, the EBIT group at Max-Planck-Institut für Kernphysik (MPIK) has recently developed and implemented the portable FLASH-EBIT [17, 18] which can be attached to beamline experiments at third and fourth generation advanced light sources and study problems involving photo-absorption. The MPIK group has developed and implemented several diagnostics that open new measurement capabilities. Notable is an ion extraction system that makes the FLASH-EBIT a powerful tool for conducting photoionization experiments [19]. In collaboration with the LLNL EBIT group and NASA/GSFC, the FLASH-EBIT has been outfitted with a complete set of grating and solid state spectrometers, including the XRS/ECS first used at LLNL [20, 21] (see figure 2). The FLASH-EBIT has already been used 
successfully at the FLASH free electron laser (FEL) [17], BESSY-II [19], the LCLS FEL [22] and Petra-III [23].

\section{Experiments involving M-shell Fe ions}

Measurements of x-ray emission and absorption from L-shell transitions in $\mathrm{M}$-shell ions have been conducted using both EBIT-I at Livermore and using the FLASH-EBIT at BESSY-II and at the LCLS FEL. Focused measurements on these transitions began in order to address the effect of Fe XVI emission that blends with $\mathrm{Fe} \mathrm{L}$ shell lines, specifically, the effect on the neon-like Fe XVII emission [13, 24]. Early measurements showed that an Fe XVI satellite line blends with the relatively strong intercombination Fe XVII line at 15.26 $\AA$, known as three dimensional. This blend must be taken into account when interpreting high resolution spectra, especially in cooler sources where significant amounts of Fe XVI are present [25]. More recently, experiments have been conducted to address large discrepancies in the predicted velocity of the M-shell ions in active galactic nuclei as a result of disagreement among theoretical values of their rest wavelengths. Wavelengths of the absorption features in $\mathrm{Fe}^{13+}-\mathrm{Fe}^{15+}$ were measured at BESSY-II by Simon et al [19] and Beilmann et al [26]. Wavelengths calculated using the relativistic many body perturbation theory (RMBPT) of Gu [11] were found to agree best with these measurements. Using the laboratory-tested values, it was found that, in the case of NGC 3783, the Fe M-shell ions have the same velocity structure as the more highly charged $\mathrm{Fe}^{16+}$ and $\mathrm{O}^{6+}$ and $\mathrm{O}^{7+}$ ions.

High resolution measurements at EBIT-I of the L-shell emission from M-shell ions have also been completed and compared with new multi-reference Møller-Plesset (MRMP) calculations $[27,28]$. For transitions measured with both EBIT-I and at BESSY-II, agreement is very good. Similarly, the wavelengths predicted by the new MRMP agree with the RMBPT of Gu et al [11] in almost every case. Using their new measurements, Beiersdorfer et al [27, 28] identified several new features in the Chandra spectrum of Capella, showing that the $\mathrm{x}$-ray emission from $\mathrm{M}$-shell ions can produce a significant amount of flux which is not included in the standard astrophysical spectral models.

\section{Summary}

Laboratory astrophysics experiments continue to play a crucial role in the interpretation of spectral data from celestial sources. With the launch of Astro-H in 2014, the first high resolution, high accuracy spectra of the iron- $\mathrm{K}$ band will become available. These will drive the need for more laboratory measurements of both the photon emission and absorption of K-shell transitions in highly charged iron and iron-group elements.

\section{Acknowledgments}

This work was partially performed under the auspices of the U S Department of Energy by Lawrence Livermore National Laboratory under contract no. DE-AC52-07NA27344 and was also supported by NASA grants to LLNL, Stanford University and the NASA/GSFC.

\section{References}

[1] Takahashi T et al 2013 Proc. SPIE $773277320 \mathrm{Z}$

[2] Mitsuda K et al 2013 Proc. SPIE 7732773211

[3] Porter F S et al 2013 Proc. SPIE 7732 77323J

[4] Beiersdorfer P 2003 Astron. Astrophys. Rev. 41 343-90

[5] Beiersdorfer P 2008 Can. J. Phys. 86 1-10

[6] Porter F S et al 2008 Rev. Sci. Instrum. 79 10E307

[7] Porter F S et al 2009 13th Int. Conf. on Low Temperature Detectors (American Institute of Physics Conf. Ser. vol 1185) ed B Young, B Cabrera and A Miller (New York: AIP) pp 454-7

[8] Beiersdorfer P, Schweikhard L, López-Urrutia J C and Widmann K 1996 Rev. Sci. Instrum. 67 3818-26

[9] Knapp D A et al 1993 Phys. Rev. A 47 2039-46

[10] Holczer T, Behar E and Kaspi S 2005 Astrophys. J. 632 788-92

[11] Gu M F, Holczer T, Behar E and Kahn S M 2006 Astrophys. J. 641 1227-32

[12] Beiersdorfer P and Lepson J K 2012 Astrophys. J. Suppl. Ser. 20128

[13] Brown G V, Beiersdorfer P, Chen H, Chen M H and Reed K J 2001 Astrophys. J. Lett. 557 L75-8

[14] Beiersdorfer P et al 2003 Science 300 1558-9

[15] Frankel M et al 2009 Astrophys. J. 702 171-7

[16] Hell N et al 2013 Phys. Scr. T156 014008

[17] Epp S W et al 2007 Phys. Rev. Lett. 98183001

[18] Epp S W et al 2010 J. Phys. B: At. Mol. Phys. 43194008

[19] Simon M C et al 2010 Phys. Rev. Lett. 105183001

[20] Porter F S et al 2004 Rev. Sci. Instrum. 753772

[21] Porter F S et al 2008 Can. J. Phys. 86 231-40

[22] Bernitt S et al 2012 Nature 492225

[23] Rudolph J K et al 2013 Astrophys. J. at press

[24] Graf A, Beiersdorfer P, Brown G V and Gu M F 2009 Astrophys. J. 695 818-24

[25] Brickhouse N S and Schmelz J T 2006 Astrophys. J. Lett. 636 L53-6

[26] Beilmann C et al 2013 Astrophys. J. at press

[27] Beiersdorfer P, Diaz F and Ishikawa Y 2012 Astrophys. J. 745167

[28] Beiersdorfer P, Lepson J K, Díaz F, Ishikawa Y and Träbert E 2013 Phys. Scr. T156 014007 